\title{
"Post-Decompressive Neuropathy": New-Onset Post-Laminectomy Lower Extremity Neuropathic Pain Different from the Preoperative Complaint
}

\author{
Lorraine A. T. Boakye, Mitchell S. Fourman, Nicholas T. Spina, Dann Laudermilch, Joon Y. Lee \\ Division of Spine Surgery, Department of Orthopedic Surgery, University of Pittsburgh Medical Center, Pittsburgh, PA, USA
}

\begin{abstract}
Study Design: Level III retrospective cross-sectional study.
Purpose: To define and characterize the presentation, symptom duration, and patient/surgical risk factors associated with 'postdecompressive neuropathy (PDN).'

Overview of Literature: PDN is characterized by lower extremity radicular pain that is 'different' from pre-surgical radiculopathy or claudication pain. Although it is a common constellation of postoperative symptoms, PDN is incompletely characterized and poorly understood. We hypothesize that PDN is caused by an intraoperative neuropraxic event and may develop early (within 30 days following the procedure) or late (after 30 days following the procedure) within the postoperative period.

Methods: Patients who consented to undergo lumbar laminectomy with or without an instrumented fusion for degenerative lumbar spine disease were followed up prospectively from July 2013 to December 2014. Relevant data were extracted from the charts of the eligible patients. Patient demographics and surgical factors were identified. Patients completed postoperative questionnaires 3 weeks, 3 months, 6 months, and 1 year postoperatively. Questions were designed to characterize the postoperative pain that differed from preoperative pain. A diagnosis of PDN was established if the patient exhibited the following characteristics: pain different from preoperative pain, leg pain worse than back pain, a non-dermatomal pain pattern, and nocturnal pain that often disrupted sleep. A Visual Analog Scale was used to monitor the pain, and patients documented the effectiveness of the prescribed pain management modalities. Patients for whom more than one follow-up survey was missed were excluded from analysis.

Results: Of the 164 eligible patients, $118(72.0 \%)$ completed at least one follow-up survey at each time interval. Of these eligible patients, 91 (77.1\%) described symptoms consistent with PDN. Additionally, 75 patients (82.4\%) described early-onset symptoms, whereas 16 reported symptoms consistent with late-onset PDN. Significantly more female patients reported PDN symptoms (87\% vs. $69 \%, p=0.03$ ). Patients with both early and late development of PDN described their leg pain as an intermittent, constant, burning, sharp/stabbing, or dull ache. Early PDN was categorized more commonly as a dull ache than late-onset PDN (60\% vs. $31 \%, p=0.052$ ); however, the difference did not reach statistical significance. Opioids were significantly more effective for patients with early-onset PDN than for those with late-onset PDN ( $85 \%$ vs. $44 \%, p=0.001)$. Gabapentin was most commonly prescribed to patients who cited no resolution of symptoms ( $70 \%$ vs. $31 \%, p=0.003$ ). Time to symptom resolution ranged from within 1 month to 1 year. Patients' symptoms were considered unresolved if symptoms persisted for more than 1 year postoperatively. In total, $81 \%$ of the patients with earlyonset PDN reported complete symptom resolution 1 year postoperatively compared with $63 \%$ of patients with late-onset PDN ( $p=0.11$ ). Conclusions: PDN is a discrete postoperative pain phenomenon that occurred in $77 \%$ of the patients who underwent lumbar laminectomy with or without instrumented fusion. Attention must be paid to the constellation and natural history of symptoms unique to PDN to effectively manage a self-limiting postoperative issue.
\end{abstract}

Keywords: Laminectomy; Spinal stenosis; Neuropathic pain; Analgesics

Received Aug 23, 2017; Revised Mar 4, 2018; Accepted Apr 25, 2018

Corresponding author: Joon Y. Lee

Department of Orthopedic Surgery, University of Pittsburgh Medical Center, Kaufmann Medical Building, Suite 1010, 3471 Fifth Ave, Pittsburgh, PA 15213, USA

Tel: +1-412-605-3218, Fax: +1-412-605-3269, E-mail: leejy3@upmc.edu 


\section{Introduction}

Currently, the best-known treatment for severe lumbar stenosis refractory to non-operative treatment is decompressive laminectomy with or without fusion. It is important for the surgeon to convey the possible and likely suboptimal outcomes to the patient during the informed consent process. Common complications of decompressive laminectomies include a prolonged inpatient stay, dural tear, wound infection, urinary tract infection, pneumonia, perioperative hematoma, deep vein thrombosis, and pulmonary embolus [1]. Additional risk factors include injuries to the nerves and the surrounding structures, incomplete resolution of preoperative symptoms, and postoperative pain.

An important distinction should be made between persistent preoperative symptoms and the evolution of a different pathology when addressing post laminectomy pain. In our experience, several patients who were seen for postoperative follow-up describe a new onset radicular pain that is different in character and nature from their preoperative pain. We termed this 'post-decompressive neuropathy' (PDN) because it is critical to understand the treatment modalities required for the optimal management of this constellation of symptoms and to assess the long-term prognostic significance of PDN for patients.

We defined and characterized the incidence, location, severity, duration, and patient/surgical risk factors associated with PDN with a prospectively administered, postoperative pain survey. This survey was designed to assess the unique characteristics and physiology of this pain syndrome. We used the Visual Analog Scale (VAS) as a portion of the survey as a means of providing objective, quantifiable date that lent itself to temporal analysis.

\section{Materials and Methods}

The study was approved by the Institutional Review Board at the University of Pittsburgh Medical Center (IRB approval no., PRO12090400) of University of Pittsburgh Medical Center; patients who consented to undergo a lumbar laminectomy with or without instrumented fusion for degenerative lumbar spine disease were prospectively followed up from July 2013 to December 2014. Patients with principal or secondary diagnoses of infection, trauma, or malignancy were excluded. Each surgery was performed by one of three fellowship trained spine sur-
Table 1. Cohort characteristics $(n=118)$

\begin{tabular}{lc} 
Characteristic & Value \\
Patient demographics & \\
Males & $64(54)$ \\
\hline Age $(\mathrm{yr})$ & $58.0 \pm 11.9$ \\
\hline $\mathrm{BMI}\left(\mathrm{kg} / \mathrm{m}^{2}\right)$ & $31.2 \pm 5.6$ \\
\hline Obese $\left(\mathrm{BMl}>30 \mathrm{~kg} / \mathrm{m}^{2}\right)$ & $64(54)$ \\
\hline Morbidly obese $\left(\mathrm{BMl}>40 \mathrm{~kg} / \mathrm{m}^{2}\right)$ & $10(8.4)$ \\
\hline Diabetes & $22(18.6)$ \\
Surgical intervention & \\
\hline \# Laminectomy levels & $3.6 \pm 1.2$ \\
\hline \# Fusion levels & $1.5 \pm 1.8$ \\
\hline Instrumentation & $75(63)$ \\
\hline Revision procedure & $32(27)$ \\
\hline Use of morphogenic protein & $4(3.4)$ \\
\hline Complications & $18(15.2)$ \\
\hline Dural tear & $1(0.8)$ \\
\hline Pulmonary embolus & 0 \\
\hline Infection & \\
\hline
\end{tabular}

Values are presented as number (\%) or mean \pm standard deviation. $\mathrm{BMI}$, body mass index.

geons at the same academic institution. All patients were consulted at their first postoperative visit. Patients for whom only one postoperative survey was completed were excluded (46 patients) (Table 1).

Patients completed the postoperative questionnaires 3 weeks, 3 months, 6 months, and 1 year postoperatively. All surveys were completed independently by each patient. The questions were designed to characterize postoperative pain (Appendix 1). Patients were able to provide subjective and objective assessments of pain. The VAS was used to establish pain burden and to monitor pain progression. Careful attention was paid to the constellation of symptoms that characterized the temporal evolution of 'new' neuropathic pain, defined as a distributional pain complaint that was not observed or recorded preoperatively and was not the indication for the decompressive procedure. PDN was diagnosed when the patient exhibited the following four characteristics: (1) lower extremity pain different from the preoperative complaint (question 3), (2) leg pain that was worse than back pain (question 2), (3) a non-dermatomal pain pattern (question 4), and (4) nocturnal pain that often disrupted sleep (question 5). Patients were offered a chance to comment on the progress 
Table 2. Group demographics and surgical characteristics for early versus late PDN

\begin{tabular}{|c|c|c|c|c|}
\hline Characteristic & No pain $(\mathrm{n}=27)$ & Early PDN (n=75) & Late PDN (n=16) & $p$-value \\
\hline Age (yr) & $56.5 \pm 12.3$ & $57.4 \pm 11.7$ & $60.5 \pm 11.2$ & 0.55 \\
\hline Sex (male:female) & $20: 7$ & $35: 40$ & $10: 7$ & 0.06 \\
\hline $\mathrm{BMI}\left(\mathrm{kg} / \mathrm{m}^{2}\right)$ & $30.1 \pm 3.6$ & $31.5 \pm 6.4$ & $31.7 \pm 4.6$ & 0.49 \\
\hline Obese $\left(\mathrm{BMl}>30 \mathrm{~kg} / \mathrm{m}^{2}\right)$ & 14 & 40 & 10 & 0.53 \\
\hline Morbidly obese $\left(\mathrm{BMl}>40 \mathrm{~kg} / \mathrm{m}^{2}\right)$ & 0 & 9 & 1 & 0.40 \\
\hline \# Laminectomy levels & $3.3 \pm 1.1$ & $3.6 \pm 1.2$ & $3.7 \pm 1.6$ & 0.53 \\
\hline \# Fusion levels & $1.6 \pm 2.2$ & $1.4 \pm 1.7$ & $1.4 \pm 1.7$ & 0.94 \\
\hline Instrumentation & 15 & 48 & 12 & 0.32 \\
\hline Revision procedure & 8 & 20 & 4 & 0.97 \\
\hline Diabetic & 4 & 14 & 4 & 0.80 \\
\hline Dural tear & 3 & 13 & 2 & 0.71 \\
\hline Bone morphogenic protein used in fusion & 1 & 3 & 0 & 0.71 \\
\hline Pulmonary embolus & 0 & 1 & 0 & 0.32 \\
\hline
\end{tabular}

Values are presented as mean \pm standard deviation or number.

PDN, post-decompressive neuropathy; BMI, body mass index.

of their pain management at subsequent appointments.

A retrospective medical record review was performed for all the patients who fulfilled the study inclusion criteria to correlate their demographic characteristics, comorbidities, surgical factors, and perioperative complications with the occurrence of PDN.

The primary outcome measures included time until resolution, modalities considered effective in PDN treatment, modalities associated with unresolved PDN, nature and character of leg pain, as well as the progression of the VAS score over time.

Statistical analyses were performed by the authors using Prism ver. 7.0 (GraphPad, La Jolla, CA, USA). Fisher's exact test was used to compare the categorical variables. Patients with both early and late-onset PDN were compared to those who remained asymptomatic. We compared those patients with early and late pathological development. In all cases, $p<0.05$ was considered statistically significant.

\section{Results}

Of the 164 eligible patients who had completed at least one follow-up survey, 118 (72\%) completed more than one survey. The average length of follow-up in this group was $196 \pm 112$ days. Seventy-five patients (46\%) reported symptoms characteristic of PDN in their initial question- naire administered at 3 weeks postoperatively. The onset of symptoms within 30 days postoperatively was considered to indicate early-onset PDN. The early-onset PDN group included 40 women with a mean age of $57.4 \pm 11.7$ years. Forty patients were obese (body mass index [BMI] $>30 \mathrm{~kg} / \mathrm{m}^{2}$ ), and nine of these patients were classified as morbidly obese $\left(\mathrm{BMI}>40 \mathrm{~kg} / \mathrm{m}^{2}\right)$. Fourteen patients had coexisting type II diabetes. Forty-eight patients underwent instrumented fusion, and 20 underwent revision procedures. Sixteen patients reported symptoms characteristic of PDN at 30 days postoperatively, defined here as lateonset PDN. Of these patients, seven were women and had a mean age of $60.5 \pm 11.2$ years. Ten patients were obese (one with BMI $>40 \mathrm{~kg} / \mathrm{m}^{2}$ ), and four had type II diabetes. Twelve patients underwent instrumented fusions, and four underwent revision surgeries (Table 2).

The proportion of women who developed PDN was significantly higher than those who did not (52\% versus $26 \%$, $p=0.03$ ) (Table 3 ). There were no statistically significant differences in the age, BMI, incidence of diabetes, mean number of laminectomy or fusion levels, use of instrumentation, use of bone morphogenic protein (BMP), or the incidence of intraoperative dural tears in the patients with and without PDN. There was no significant difference between the number of levels included in the fusion or whether the surgery was a revision procedure amongst asymptomatic patients or patients who developed early- 
Table 3. Patient demographics and surgical characteristics of developing PDN

\begin{tabular}{|c|c|c|c|}
\hline Characteristic & No PDN (n=27) & PDN (n=91) & $p$-value \\
\hline Age (yr) & $56.6 \pm 12.3$ & $57.9 \pm 11.6$ & 0.59 \\
\hline Sex (male:female) & $20: 7$ & $44: 47$ & $0.03^{*}$ \\
\hline $\mathrm{BMI}\left(\mathrm{kg} / \mathrm{m}^{2}\right)$ & $30.1 \pm 3.6$ & $31.6 \pm 6.1$ & 0.24 \\
\hline Obese $\left(\mathrm{BMl}>30 \mathrm{~kg} / \mathrm{m}^{2}\right)$ & 14 & 50 & 0.83 \\
\hline Morbidly obese $\left(\mathrm{BMI}>40 \mathrm{~kg} / \mathrm{m}^{2}\right.$ ) & 0 & 10 & 0.11 \\
\hline \# Laminectomy levels & $3.3 \pm 1.1$ & $3.6 \pm 1.3$ & 0.26 \\
\hline \# Fusion levels & $1.6 \pm 2.2$ & $1.4 \pm 1.7$ & 0.75 \\
\hline Instrumentation & 15 & 60 & 0.37 \\
\hline Revision procedure & 8 & 24 & 0.81 \\
\hline Diabetic & 4 & 18 & 0.78 \\
\hline Dural tear & 3 & 15 & 0.76 \\
\hline Morphogenic protein used in fusion & 1 & 3 & $>0.99$ \\
\hline
\end{tabular}

Values are presented as mean \pm standard deviation or number. PDN, post-decompressive neuropathy; BMI, body mass index. * $p<0.05$.

Table 4. Nature of leg complaint

\begin{tabular}{lccc} 
Variable & $\begin{array}{c}\text { Early PDN } \\
(\mathrm{n}=75)\end{array}$ & $\begin{array}{c}\text { Late PDN } \\
(\mathrm{n}=16)\end{array}$ & $p$-value \\
Intermittent & 37 & 10 & 0.41 \\
Constant & 27 & 5 & 0.78 \\
Burning & 20 & 6 & 0.38 \\
Sharp/stabbing & 27 & 4 & 0.56 \\
\hline Dull ache & $45(60)$ & $5(31.3)$ & 0.052 \\
\hline
\end{tabular}

Values are presented as number or number (\%).

PDN, post-decompressive neuropathy.

or late-onset PDN (Table 2). Revision procedures did not predispose a patient to PDN. Compared to late PDN, early PDN was more likely to be categorized as a dull ache; however, this difference was not significant (60\% versus $31 \%, p=0.052$ ) (Table 4). There was no significant difference in the characterization of leg pain between early- and late-onset PDN patients (Table 5).

Time until PDN symptom resolution ranged from within 1 month to 1 year. Patients were considered to have persistent PDN if their symptoms persisted for more than 1 year postoperatively. Complete resolution of symptoms was reported by $81 \%$ of those with early PDN and $63 \%$ of those with late PDN at 1 year postoperatively $(p=0.11$ ). Total 20 patients, 14 of 75 (19\%) with early PDN and 6 of 16 (38\%) with late PDN never experienced complete symptom resolution as per the patient-reported survey re-
Table 5. Character of leg pain

$\begin{array}{lccr}\text { Variable } & \begin{array}{c}\text { Early PDN } \\ (\mathrm{n}=75)\end{array} & \begin{array}{c}\text { Late PDN } \\ (\mathrm{n}=16)\end{array} & p \text {-value } \\ \text { Worse at night } & 24(32) & 6(37.5) & 0.77 \\ \text { Wakes up from sleep } & 31(41.3) & 6(37.5) & >0.99 \\ \text { Worsened by activity } & 20(26.7) & 7(43.8) & 0.23\end{array}$

Values are presented as number (\%).

PDN, post-decompressive neuropathy.

Table 6. Time to resolution of leg pain

\begin{tabular}{lccc} 
Variable & $\begin{array}{c}\text { Early PDN } \\
(\mathrm{n}=75)\end{array}$ & $\begin{array}{c}\text { Late PDN } \\
(\mathrm{n}=16)\end{array}$ & $p$-value \\
Within $1 \mathrm{mo}$ & 22 & - & Not applicable \\
Within $3 \mathrm{mo}$ & 47 & 8 & 0.40 \\
Within $6 \mathrm{mo}$ & 56 & 10 & 0.36 \\
Within $1 \mathrm{yr}$ & 61 & 10 & 0.11 \\
\hline Never resolved & $14(18.7)$ & $6(37.5)$ & 0.11
\end{tabular}

Values are presented as number or number (\%).

PDN, post-decompressive neuropathy.

sponses. Early PDN resolved within 1 month in 22 out of 75 patients (29\%), improved within 3 months in 47 (63\%), resolved within 6 months in 56 (75\%), and alleviated within 1 year postoperatively in 61 of the patients (81\%) (Fig. 1). There was no statistically significant difference between the rate of symptom resolution or time to symp- 

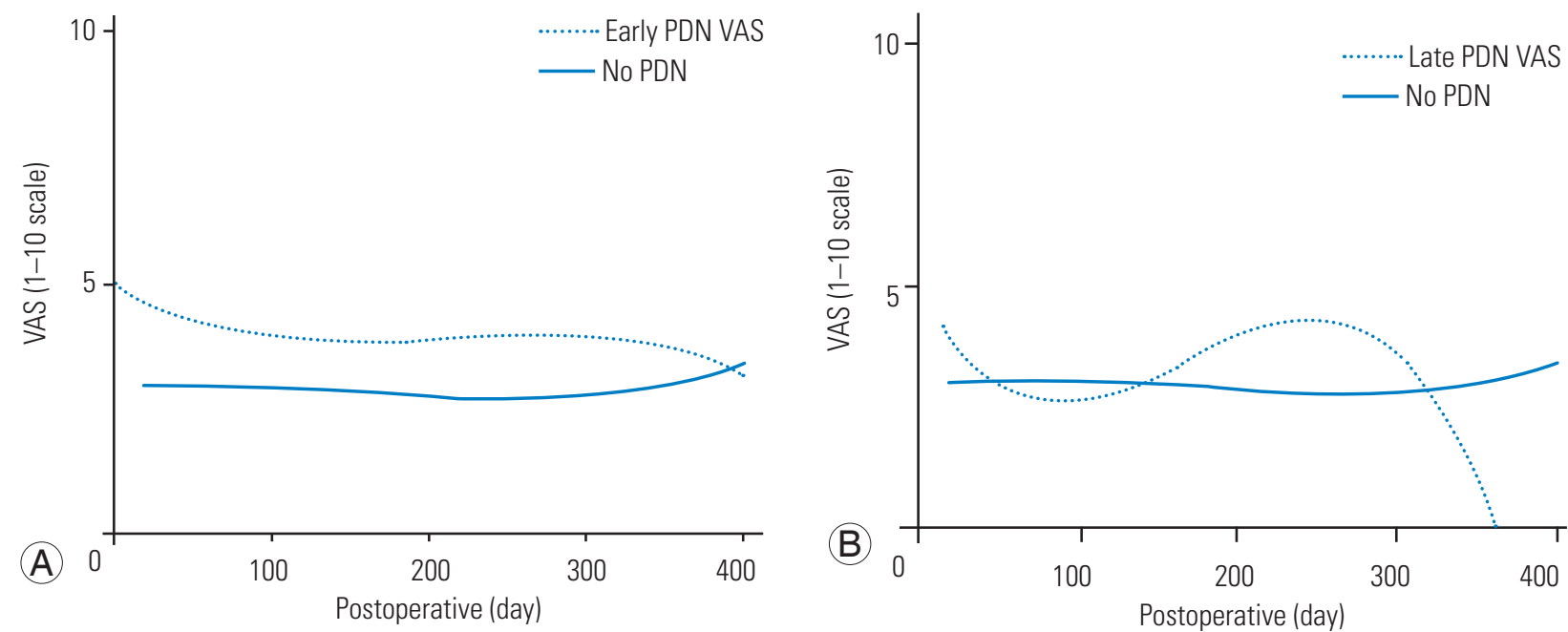

Fig. 1. Evaluation of leg pain in patients with early versus late PDN. VAS score over time early PDN versus no PDN. PDN, post-decompressive neuropathy; VAS, Visual Analog Scale.

Table 7. Pain control modalities most associated with resolution of symptoms as described by patients

\begin{tabular}{lccc} 
Variable & Early PDN $(\mathrm{n}=75)$ & Late PDN (n=16) & $p$-value \\
Medrol dose pak & 12 & 1 & 0.45 \\
Physical therapy & 40 & 11 & 0.28 \\
\hline Neurontin & 29 & $7(43.8)$ & 0.78 \\
Opioids & $64(85.3)$ & 7 & $0.001^{*}$ \\
\hline Non-steroidal anti-inflammatory drugs & 38 & 0.78 \\
\hline
\end{tabular}

Values are presented as number or number (\%).

PDN, post-decompressive neuropathy.

* $p<0.05$.

Table 8. Pain control modalities most associated with unresolved symptoms as described by patients

\begin{tabular}{lccc} 
Variable & PDN resolved $(\mathrm{n}=71)$ & Unresolved PDN (n=20) & $p$-value \\
Medrol dose pak & 8 & 5 & 0.15 \\
\hline Physical therapy & 38 & 13 & 0.45 \\
\hline Neurontin & $22(31.0)$ & $14(70.0)$ & $0.003^{*}$ \\
Opioids & 56 & 18 & 0.34 \\
Non-steroidal anti-inflammatory drugs & 34 & 11 & 0.62 \\
\hline
\end{tabular}

Values are presented as number or number (\%).

PDN, post-decompressive neuropathy.

" $p<0.05$.

tom resolution between early and late PDN groups (Table $6)$. Opioids were more effective in patients with early onset $\mathrm{PDN}$ than in those with late onset PDN (85\% versus $44 \%, p=0.001$ ) (Table 7). Gabapentin was most commonly cited as an attempted treatment method among patients with PDN whose symptoms did not resolve (70\% versus
$31 \%, p=0.003)$ (Table 8).

\section{Case example}

A 56-year-old man with a previous history of a prior microdiscectomy at L4-5 (motor strength graded at 4/5). 


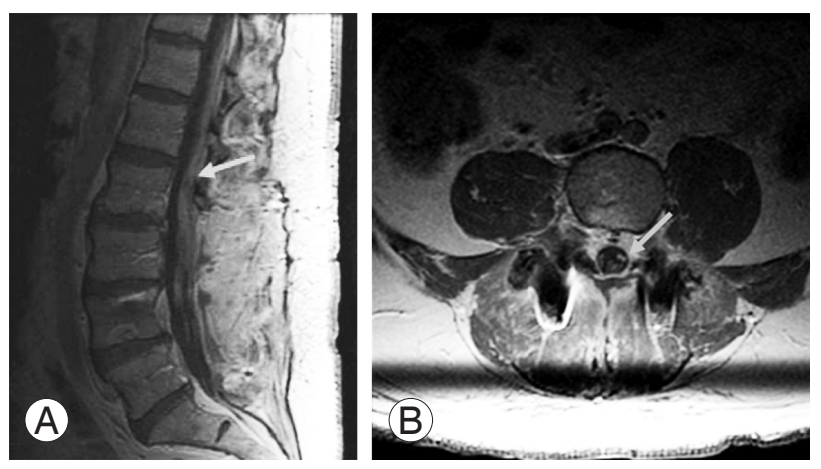

Fig. 2. Magnetic resonance imaging with gadolinium contrast showing enhancement of cauda equina (arrows). (A) Axial view. (B) Sagittal view.

Magnetic resonance imaging (MRI) demonstrated congenital spinal stenosis from L2-5, with a recurrent herniated disc at L4-5. The patient's radiographic imaging was consistent with L4-5 spondylolisthesis. After failing nonoperative management, the patient underwent a L2-5 revision laminectomy with a L4-5 posterolateral instrumented fusion. The surgical and immediate postoperative course was uneventful, and the patient was discharged on postoperative day 3 .

Two weeks postoperatively, the patient followed up in clinic and endorsed severe bilateral leg pain that was different in nature from his preoperative unilateral (left) leg pain. His preoperative L5 radiculopathy and foot drop had improved significantly; however, the patient now endorsed non-dermatomal pain and paresthesias in both legs. The symptoms worsened at night and were strong enough to wake him from sleep; they were only partially relieved by ambulation. He did not report any fevers, chills, or other constitutional symptoms. His symptoms were purely sensory, with no motor involvement.

An MRI with gadolinium contrast revealed an enhancing cauda equina, indicative of significant endoneural edema and a leaky blood brain barrier (Fig. 2). The patient lacked clinical manifestations that suggested common postoperative complications or a separate disease process, such as spinal meningitis, Guillain-Barre Syndrome, and chronic inflammatory polyneuropathy. As the aforementioned conditions were essentially ruled out, which helped to support the alternative diagnosis of PDN. The patient returned to clinic for continued monitoring and reassurance. He was medically managed with a short course of steroids (1 week) and high dose gabapentin $(1,200 \mathrm{mg}$, twice daily) for 3 months, during which time the patient noted reduction and eventual relief from pain. Gabapen- tin was gradually weaned down over 5 months; by this time, the patient's symptoms had resolved.

\section{Discussion}

The primary aim of this study was to name and characterize a common constellation of clinical symptoms after lumbar laminectomy with or without instrumented fusion. Our data suggest that a considerable proportion (77\%) of our patients developed severe neuropathic pain that differed from their preoperative indication. Optimal management was limited by an elusive diagnosis. PDN diagnosis was established following careful assessment of patient-reported symptoms and the results of prospective survey analysis (including the VAS). A combination of the targeted questionnaire and validated outcome score was used to highlight the risk factors for and natural history of the pathology. Diagnosis of PDN was only conferred after careful exclusion of more commonly occurring postoperative pathologies.

The initial mention of 'new' postoperative pain ranged from several days to weeks after surgery with bimodal distribution, presenting early (within 30 days postprocedure) or late (more than 30 days post-procedure). In our experience, PDN can be debilitating for the patients because the symptoms may last from several weeks to months. Despite being a commonly occurring condition, the postoperative pain phenomenon has evaded precise characterization and protocol for optimal management.

PDN is characterized by the delayed onset (usually several days to weeks) of new lower extremity neuropathic pain or paresthesia in a non-dermatomal distribution following a lumbar laminectomy and/or fusion procedure. PDN pain commonly worsens at nighttime (significant enough to wake patients from sleep). PDN is not exacerbated by postural changes and is often partially relieved by walking. Symptoms are purely sensory without dermatomal distribution. There is no motor involvement. The symptoms characters remained constant throughout the follow-up period; thus, the phenomenon was further distinguished by the lack of symptom progression.

Several prior reports note a post-laminectomy pain syndrome; however, without significant sample size or organization to propose an independent diagnosis, PDN may be distinguished from 'post laminectomy syndrome (PLS), or 'failed back surgery syndrome (FBSS)', a catastrophic 
complication of lumbar laminectomy. PLS or FBSS is defined as "lumbar spinal pain of unknown origin either persisting despite surgical intervention or appearing after surgical intervention for spinal pain originally in the same topographical location [2,3]." Unlike PLS, PDN symptoms differ from the preoperative issues and often resolve within 3-6 months with only supportive care. Further, PDN is purely a lower extremity phenomenon. Therefore, the presence of back pain may be more indicative of PLS.

Rowan et al. [4] demonstrated an association between BMP use during spinal fusions with posteriorly inserted interbody cages and the incidence of postoperative radiculitis. The authors proposed that the mechanism for this new pain was an excessive inflammatory response and ectopic bone formation [5-9]. In a cohort-controlled study, Lykissas et al. [10] found a significantly higher rate of persistent postoperative neurologic (motor and sensory) deficit and pain in patients who had received rh-BMP-2. However, in contrast to this BMP-associated radiculitis, PDN is most severe at nighttime, is purely sensory, and has non-dermatomal in distribution. Our results further suggest that PDN is not associated with BMP use.

Significantly more female patients reported PDN symptoms. Ineffective pain control in women has been recognized as a factor for the different postoperative outcomes across sexes [11]. Shabat et al. [12] postulated that sex-related differences in the patient-reported outcome following lumbar spine decompression might be attributable to the difference in the hormone levels of the sexes that predispose them to differences in the pain perception despite similar prescribed pain control regimens. Female patients are known to experience less pain relief and were less satisfied with the postoperative results [12]. In a benchtop study, Cicero et al noted sex-based differences in the pain response despite the opioid dose being doubled in women. The authors concluded that the sex differences in pain response were likely secondary to inherent differences in brain sensitivity rather than pharmacokinetics $[11,13]$. Women undergo lumbar laminectomies at later stages of the disease; therefore likely imparting a lower preoperative functional status that negatively affects postoperative recovery [14].

The pathophysiology of PDN is unclear. Imaging results obtained in the workup of the cited case example showed enhancement of the cauda equine on contrast-MRI. It is possible that surgical trauma to the cauda equina or dorsal root ganglion initiated an inflammatory cascade simi- lar to a demyelinating process. Hugon et al. [15] hypothesized that excitatory neurotransmitters exert neurotoxic effects on the peripheral nerves, leading to endoneural edema and demyelination. L-glutamate and L-aspartate cause excitation by binding to the N-methyl-D-aspartate receptor and likely cause toxic effects (demyelination and resultant neuropathy) through this mechanism. We hypothesize that endoneural damage with partial demyelination may be the underlying pathophysiology of PDN. However, without a large body of correlative imaging, this theory needs further validation. Reduction in the PDN symptoms may correspond with the resolution of this demyelinating process. Symptoms may be attributable to the classic localized 'epineural edema and nerve strangulation,' considering the absence of symptom progression associated with nerve compression that typically manifests with both sensory and motor components [16,17].

The limitations of our study include the inherent subjectivity of patient-reported outcomes. Neuropathy was used as a generic term to describe a neurogenic, distributional symptom. However, histological examination was not possible for these patients because we observed a postoperative phenomenon. However, future work may consider additional nerve studies, such as magnetic resonance neurography, that would enable the objective characterization of the symptoms. As is the case with several other pain related syndromes, there may be psychogenic causes associated with the development and characterization of the symptoms. We did not consider the primary diagnoses of mental health disorders or the duration of preoperative symptoms in our analysis. We isolated the symptoms most unique to the specific set of factors that appeared to indicate PDN. Our analysis is also limited by the lack of an accepted outcome measure designed to identify the patients with PDN. Although our questionnaire was designed to capture PDN, it has not been previously validated in studies with a large sample size. The questionnaire was designed to highlight the differences between usual postoperative pain complaints and this postoperative pain phenomenon that has not yet been described. These patients were selected because of pain complaints that appeared to be radicular in nature. We believe that it is more likely that these patients' complaints are neuropathic because they were remote from the surgical site and were distributional. However, the accuracy of our data may have been affected by the subjectivity of this complaint; therefore, a larger sample size is needed to 
validate our incidence rate. Pain level was assessed at different intervals throughout the study. The VAS was used to assess pain given that it is an objective and validated outcome measure. While we analyzed a large sample size during the study period, our findings suggest that this work is insufficient to fully characterize PDN. Future work will continue using the same metric to capture a larger sample of patients and focus on capturing mid- and longterm outcomes of patients previously treated for PDN.

\section{Conclusions}

This study presents the initial characterization of PDN, a largely self-limiting condition that occurred in approximately $77 \%$ of the patients who underwent a lumbar laminectomy with or without instrumented fusion at our institution. Female sex was associated with PDN development, and the use of opioids was most strongly associated with symptom resolution among those with early-onset PDN (symptom reliefs within 30 days postoperatively). Further prospective studies involving a larger sample size are warranted to fully characterize PDN and describe effective treatment modalities for this condition.

\section{Conflict of Interest}

No potential conflict of interest relevant to this article was reported.

\section{References}

1. Giannadakis C, Nerland US, Solheim O, et al. Does obesity affect outcomes after decompressive surgery for lumbar spinal stenosis?: a multicenter, observational, registry-based study. World Neurosurg 2015;84:1227-34.

2. Teixeira MJ, Yeng LT, Garcia OG, Fonoff ET, Paiva WS, Araujo JO. Failed back surgery pain syndrome: therapeutic approach descriptive study in 56 patients. Rev Assoc Med Bras (1992) 2011;57:282-7.

3. Merskey H, Bogduk N; International Association for the Study of Pain. Classification of chronic pain: descriptions of chronic pain syndromes and definitions of pain terms. Seattle (WA): International Association for the Study of Pain; 1994.

4. Rowan FE, O’Malley N, Poynton A. RhBMP-2 use in lumbar fusion surgery is associated with tran- sient immediate post-operative leg pain. Eur Spine J 2012;21:1331-7.

5. MacDonald KM, Swanstrom MM, McCarthy JJ, Nemeth BA, Guliani TA, Noonan KJ. Exaggerated inflammatory response after use of recombinant bone morphogenetic protein in recurrent unicameral bone cysts. J Pediatr Orthop 2010;30:199-205.

6. Krishna M, Pollock RD, Bhatia C. Incidence, etiology, classification, and management of neuralgia after posterior lumbar interbody fusion surgery in 226 patients. Spine J 2008;8:374-9.

7. Smucker JD, Rhee JM, Singh K, Yoon ST, Heller JG. Increased swelling complications associated with offlabel usage of rhBMP-2 in the anterior cervical spine. Spine (Phila Pa 1976) 2006;31:2813-9.

8. Vaidya R, Carp J, Sethi A, Bartol S, Craig J, Les CM. Complications of anterior cervical discectomy and fusion using recombinant human bone morphogenetic protein-2. Eur Spine J 2007;16:1257-65.

9. Wong DA, Kumar A, Jatana S, Ghiselli G, Wong K. Neurologic impairment from ectopic bone in the lumbar canal: a potential complication of off-label PLIF/TLIF use of bone morphogenetic protein-2 (BMP-2). Spine J 2008;8:1011-8.

10. Lykissas MG, Aichmair A, Sama AA, Hughes AP, Lebl DR, Cammisa FP, Girardi FP. Nerve injury and recovery after lateral lumbar interbody fusion with and without bone morphogenetic protein-2 augmentation: a cohort-controlled study. Spine J 2014;14:217-24.

11. Walker JS, Carmody JJ. Experimental pain in healthy human subjects: gender differences in nociception and in response to ibuprofen. Anesth Analg 1998;86:1257-62.

12. Shabat S, Folman Y, Arinzon Z, Adunsky A, Catz A, Gepstein R. Gender differences as an influence on patients' satisfaction rates in spinal surgery of elderly patients. Eur Spine J 2005;14:1027-32.

13. Cicero TJ, Nock B, Meyer ER. Sex-related differences in morphine's antinociceptive activity: relationship to serum and brain morphine concentrations. J Pharmacol Exp Ther 1997;282:939-44.

14. Katz JN, Wright EA, Guadagnoli E, Liang MH, Karlson EW, Cleary PD. Differences between men and women undergoing major orthopedic surgery for degenerative arthritis. Arthritis Rheum 1994;37:68794. 
15. Hugon J, Vallat JM, Leboutet MJ. Cytotoxic properties of glutamate and aspartate in rat peripheral nerves: histological findings. Neurosci Lett 1987;81:16.

16. Klusakova I, Dubovy P. Experimental models of peripheral neuropathic pain based on traumatic nerve injuries: an anatomical perspective. Ann Anat 2009;191:248-59.

17. Bennett GJ, Xie YK. A peripheral mononeuropathy in rat that produces disorders of pain sensation like those seen in man. Pain 1988;33:87-107. 
Appendix 1. Postoperative questionnaire version 2

Postoperative questionnaire: (to be filled in by MA, nurse, or physician)

Surgical procedure:

Iliac crest harvest

_ Use of morphogenic protein

Follow-up visit: _ 3 week _ 3 month _ 6 month _ 1 year

\section{Patient questionnaire}

1. Are you experiencing significant pain following your procedure: (yes) (no)

2. Is this pain in your back, legs, or both: (back) (legs) (both)

3. If you're having pain in your legs, is it different than the pain you were experiencing before your surgery: (yes) (no)

4. Where is the pain in your legs, please circle where it hurts?
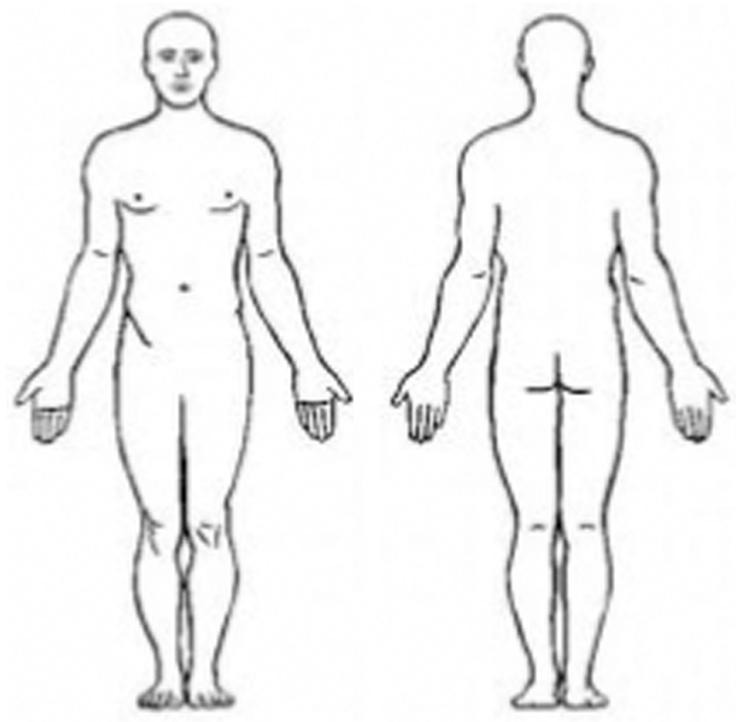

5. Please characterize the leg pain (you may circle more than one):

a. Intermittent

b. Constant

c. Burning

d. Sharp, stabbing

e. Dull ache

f. Worse at night

g. Pain that wakes you up from sleep

h. Worsened by activity

i. Other:

6. Please circle the modalities that you have tried/have been prescribed postoperatively and whether that modality has provided any relief of your leg pain symptoms.

a. Medrol dose pack: (yes) (no)

b. Physical therapy: (yes) (no)

c. Neurontin: (yes) (no)

d. Oxycodone, percocet, vicodin: (yes) (no)

e. NSAIDs (e.g., motrin, aleve, ultram): (yes) (no)

f. Other:

7. Has your leg pain/leg symptoms/tingling/ improved since your last visit

a. Yes

b. No

8. If you pain in your legs has improved or resolved, at what time frame following surgery did this occur?

a. $0-3$ weeks

b. 3 weeks to 3 months

c. 3-6 months

d. 6 months -1 year

e. It has not resolved

9. Please rate your leg pain on the Visual Analog Scale.

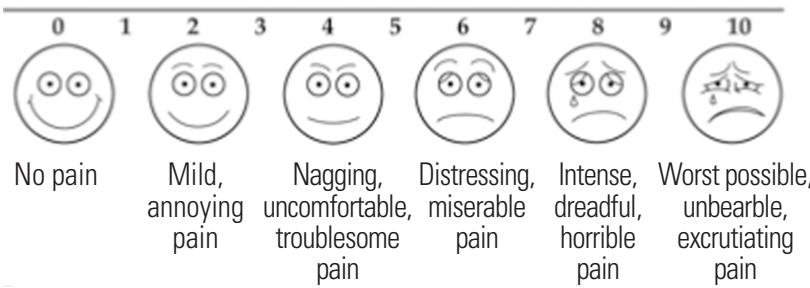

